\title{
El esplendor de la producción del libro en la Edad Media. Del manuscrito al incunable. ¿Cómo de oscura era la Edad Media?
}

\author{
Albrecht Classen \\ University of Arizona \\ aclassen@arizona.edu \\ https://dx.doi.org.10.12795/futhark.2020.il 5.03
}

Fecha de recepción: 15.04 .2020

Fecha de aceptación: 10.06.2020

\begin{abstract}
:
In this study I pursue once again the development of the medieval manuscript to the incunabulum, focusing in particular on the progress of German medieval literature. The question being pursued here focuses on how much the manuscript tradition can tell us something about the popularity of individual works. Manuscript studies thus promote a literary-historical analysis

Keywords: manuscripts, incunabula, medieval German literature, Codex Manesse, Ambraser Heldenbuch, Oswald von Wolkenstein,
\end{abstract}

\section{Título en Inglés}

\begin{abstract}
FALTA.
Keywords: FALTA.

Sumario: Introducción. I. La "oscura Edad Media" 2. El manuscrito medieval. 3. La historia de los manuscritos 4. Literatura y manuscritos. 5. Los libros de horas: el punto culminante en la producción de manuscritos medievales. 6. Codex Manesse. 7. Oswald von Wolkenstein. Conclusiones.
\end{abstract}

\section{Introducción}

Entre 1504 y 1516 , el funcionario de aduanas de Bolzano, Hans Ried, por encargo del Káiser Maximiliano I., se esfuerza en escribir a mano el que más tarde se denominará Ambraser Heldenbuch (El libro de héroes de Ambras). De esta forma crea uno de los manuscritos más influyentes de la Edad Media tardía, y esto en un

\begin{tabular}{l|l}
\hline Futhark I5 (2020) & 33
\end{tabular}


tiempo en el que hace ya mucho del predominio de la imprenta y del papel, que sustituye al pergamino, como material de escritura e impresión (Klarer). Pero pese a que desde la invención de la imprenta por Johannes Gutenberg (Füssel), aproximadamente entre 1450 y 1500 domina el incunable o libro impreso, sin embargo no sólo este manuscrito influyente sino también otros van a documentar el interés incesante que existe por estos métodos tradicionales para la transmisión de textos.

Todavía hoy perdura la fascinación por el manuscrito, pues existen numerosos documentos valiosos que siguen usando el pergamino y la escritura a mano, lo que les confiere un gran valor. Entre estos encontramos actas de nacimiento, actas fundacionales, premios, contratos, constituciones, etc. No obstante, hay que tener en cuenta que en la actualidad prácticamente todo el mundo de la impresión se basa en el papel o, lo que entretanto se ha desarrollado de forma veloz y que es indicativo de un más amplio cambio de paradigma, en el medio digital. El manuscrito de pergamino se encuentra, por tanto, entre las rarezas del mundo moderno y representa una situación excepcional con un valor sagrado. Hasta nuestros días, la torá es producida con piel de oveja y ternero, a ser posibles de los fetos, ya que esta resulta especialmente delicada y fina, y permite de forma propicia la escritura a través de plumas de pavo u otros utensilios. La elaboración de una torá constituye hasta el presente un acto por así decirlo religioso, el cual solo le está permitido a escribas o aprendices, que deben realizar su tarea de una forma fiable (https://www.myjewishlearning.com/article/torahscroll/; ver también: https://en.wikipedia.org/wiki/Sefer_Torah\#Production).

La investigación de la literatura medieval y de otros textos se remonta a varios siglos atrás, y aunque a veces se siguen produciendo nuevos hallazgos, disponemos a día de hoy de un conocimiento sólido sobre el mundo de los manuscritos de la época anterior a la Modernidad (Glenisson). Las siguientes consideraciones no sólo tienen en cuenta aspectos filológicos o paleográficos, lo que ha sido ya ampliamente tratado en el estudio de obras medievales de las que existe al menos una edición moderna, sino también en aquello que tiene que ver con la producción literaria medieval en un sentido técnico. Precisamente en esto último, que ha sido a menudo pasado por alto, quiero centrarme en esta conferencia. En concreto hablaré del papel de los manuscritos como una forma de acceso relevante a la época anterior a la Modernidad.

\section{La "oscura Edad Media"}

Por todas partes se escuchan críticas muy negativas sobre la Edad Media. A menudo, de forma ingenua y sin un conocimiento de fondo se la describe como la "oscura Edad Media" (Althoff). Por supuesto es fácil dictar juicios negativos desde 
una perspectiva histórico-cultural, pues basta con coger cualquier cuadrícula para ver cómo de forma simple sus elementos positivos se pierden entre los restantes aspectos negativos, que dominan la imagen. Sin lugar a dudas, la época anterior a la Modernidad se muestra como una época cultural que, vista desde un punto de vista técnico, está en claro retroceso con respecto a la nuestra. Muchas cosas de la vida cotidiana, que hoy nos resultan evidentes, no existían entonces. La asistencia médica y la higiene general estaban en gran medida poco desarrolladas; la percepción del mundo era geocéntrica, la creencia en Dios dominaba al hombre completamente, los conocimientos sobre países o continentes extranjeros eran muy limitados, etc.

No obstante, si se compara el siglo veinte con la Edad Media, se puede afirmar que no es hasta la Modernidad cuando acontece la verdadera barbarie. Incluso cuando oímos también que en la Edad Media hubo guerras, cruzadas, hambrunas, pestes, pogromos contra los judíos o enemistad global contra los musulmanes (siempre desde la visión de Europa), sin embargo no existe un Holocausto hasta 1933. Genocidios como el de los turcos contra los armenios no sucede hasta 1915 y en Burundi el genocidio de los Tutsis contra los hutus, que se da en tres grandes olas, no ocurre hasta 1972, 1988 y 1993, respectivamente. El número de armas de destrucción masiva, de las que disponen hoy numerosos países en el mundo, bastaría para extinguir toda la Tierra.

A la Modernidad se le trata de dar un aspecto positivo como estrategia de contraste para calificar la Edad Media de salvaje o primitiva.

Es evidente que existe el peligro de que, a través de estas consideraciones, se pudiera de manera equivocada idealizar o glorificar la Edad Media. No se trata de eso, lo que sería, desde una perspectiva objetivo-científica, completamente falso. Pero el juicio negativo general sobre la época anterior a la Modernidad resulta igualmente peligroso y erróneo. No se puede ni se debe colocar una época cultural junto a otra para compararlas, pues esto solo conduciría al uso de criterios equivocados para obtener resultados en absoluto sólidos y verificables. La electricidad, por ejemplo, pertenece a nuestro mundo moderno, pero eso no significa que la Edad Media tenga que ser automáticamente "oscura" tanto en un sentido literal como figurado.

\section{El manuscrito medieval}

A pesar de que hasta hoy solo nos habría llegado un $10 \%$ de los manuscritos que se produjeron durante la Edad Media (Haye), su ingente cantidad demuestra que nuestros valores histórico-culturales son extremadamente subjetivos. Tan solo en Francia se han documentado hasta la fecha 
aproximadamente 50.000 volúmenes de antes del 1.500 , de los cuales aproximadamente 25.000 se encuentran en la Biblioteca Nacional de París, 22.000 en bibliotecas municipales y unos 3.700 en bibliotecas universitarias. Estos quedaron reservados y fueron creados casi completamente para los intelectuales y la élite política y reflejan su formación y poder económico.

Sin embargo, nos sorprendería si nos centráramos en el mundo de los simples campesinos, que resulta por tanto un estereotipo (Rural Space); asimismo, en el caso de fijarnos en la vida de la alta nobleza, es posible que no saliéramos de nuestro asombro. Por ejemplo, las damas nobles con formación disfrutaban de unas condiciones de vida completamente distintas a las de los monjes de las distintas órdenes. Las condiciones sociales en la Edad Media eran muy amplias, lo cual nos impide poder hacer hoy generalizaciones sobre la sociedad de entonces (Rural Space).

Lo que los manuscritos nos transmiten refleja en la mayoría de los casos el nivel de formación de los mecenas, esto es, de los clérigos, a menudo obispos de alto rango o abades. Sabemos además que una buena parte de la literatura medieval en todo el mundo, así como textos religiosos de mujeres, fue impulsada por la aristocracia (Bumke), y que incluso hubo mujeres que participaron activamente como escribas en la producción de manuscritos. Esto se llevaba a cabo a menudo en monasterios femeninos (Beach). El género increíblemente floreciente de los Libros de horas, que sirvieron para la devoción en privado, demuestra una y otra vez el esplendor bibliófilo que se desarrolló en los monasterios. Aquí me detendré de nuevo más adelante a propósito de un ejemplar muy especial. Como siempre ocurre que se critican los manuscritos medievales, estos muestran sin embargo, de una forma diversa, el elevado nivel de desarrollo cultural de aquella sociedad previa a la invención de la imprenta. Con asombro incluso se puede constatar que las habilidades de los escribas de los monasterios o las cualidades de los manuscritos iluminados eran sumamente avanzados, como permiten poner ante nuestros ojos sus proezas, por ejemplo, el Libro de Kells -o Gran Evangelio de San Columba(Classen, "The Book of Kells").

Esto posibilita ofrecer una primera visión general de la historia de los manuscritos. A continuación me centraré en algunos ejemplares especiales de la Edad Media temprana y tardía, que serían representativos del esplendor y del alto nivel de desarrollo cultural de esa época. Esta incursión constituye una breve síntesis de la parte técnica de un manuscrito, que, después de numerosos procesos de tratamiento serán empleados, descritos y recogidos en un códice.

\section{La historia de los manuscritos medievales}


Hablar de manuscritos en el Medievo conlleva al mismo tiempo hacerlo de los monasterios medievales, donde únicamente existió a largo plazo una formación basada en la transmisión escrita y donde los monjes se esforzaban de manera intensiva en escribir los textos teológicos y copiarlos, fijar por escrito trabajos científicos y traducir obras de distintos campos del latín a una de las lenguas populares. A finales del siglo segundo después de Cristo, los rollos de pergamino se crean por necesidad bajo el reinado de Eumenes II de Pérgamo, porque según Plinio El Viejo, el rey egipcio Ptolomeo había bloqueado la exportación de papiro hacia Pérgamo, debido a la envidia que sentía ante la competencia de la floreciente Biblioteca y de la Ciencia en Alejandría. En el siglo cuarto o quinto los rollos de papiro y pergamino son reemplazados por el libro moderno en su fase temprana, esto es, el códice medieval, lo que da lugar a una revolución cultural, ya que permite al lector, de forma rápida y sin esfuerzo, pasar de una página a la siguiente, lo que proporciona hasta hoy una gran ventaja incluso frente al libro digital (Corsten et al., Hrsg.).

Desde la Edad Media temprana se libera el manuscrito en parte del contexto de los monasterios, ya que surgen cada vez más los scriptoria (,lugares para escribir") sobre todo relacionados con el florecimiento, a partir del siglo doce, de las universidades. En numerosos lugares se crean talleres de escritura, lo que contribuye a un enorme desarrollo de la literatura universal. La productividad de estos talleres aumenta considerablemente en la Edad Media tardía, lo que dura hasta casi dos siglos después de 1450, cuando la imprenta adquiere un amplio desarrollo o sobrepasa la producción del manuscrito, si bien este, como el ya comentado Libro de Héroes de Ambras, se continúa produciendo con carácter representativo.

Confeccionar un manuscrito precisa siempre de un grupo mayor de artesanos, expertos y artistas, ya que supone numerosos pasos individuales a seguir antes de poder obtener una lámina de pergamino idónea para escribir en ella: primero se debe elegir el animal que sea más apropiado para este objetivo. La piel de vaca o de caballo es demasiado gruesa. Las mejores para ello son las de oveja, cabra $\circ$ ternero, ya que su piel es lo suficientemente fina para poder emplearse como material de escritura. Estos animales se crían en grandes rebaños hasta que tienen el tamaño como para extraer de su piel una buena lámina. Al pergamino, que se produce de la piel de un ternero, se le denomina vellum, y al que se hace con la piel de oveja, pergamentum. Una vez que el animal es sacrificado, se le extrae la piel, que se limpia cuidadosamente por ambas partes. Se retiran los pelos y la grasa. Después se estira la piel en un marco y a continuación se mete en agua de cal para poder efectuar el tratamiento químico necesario. Luego se deja secar. Si la piel se encoje rápidamente y se vuelve dura y quebradiza, ya no es posible utilizarla. 
A continuación se repasa la piel de nuevo con un cuchillo para retirar las últimas huellas del animal. Cuando la lámina está lista, se despliega y se limpia con una piedra pómez. En el proceso siguiente se recorta el pergamino y se cierran o cosen los posibles agujeros o fisuras. Como el animal tenía que ser sacrificado, se producía siempre un agujero por la punción. Seguidamente, el rubricador dibuja las líneas en las que pinta el escriba. Un ilustrador adorna las iniciales o añade ilustraciones en los márgenes de la página del texto o en toda la página. Después hay un corrector que comprueba el texto y lo mejora en caso necesario con una navaja de afeitar. Solo entonces se unen las distintas hojas con tablillas, en la parte anterior y posterior, y hebillas de sujeción.

El papel de los manuscritos se reutilizaba, a menudo cuando se consideraba que el texto que contenía ya no era correcto o adecuado. Entonces se raspaba y se reescribía en ellos, pero como la tinta penetraba en el pergamino, siempre se quedaba una huella visible del texto original (un fenómeno que se denomina palimpsesto) lo cual hoy nos permite, con ayuda de una técnica óptica, reconstruir el texto antiguo. Se recurría a este procedimiento debido a que el pergamino era muy costoso para producirlo en grandes cantidades y no se podía tirar simplemente por el valor que tenía (Eisenlohr; Becker). Solo cuando se disponía de un mayor rebaño de ganado y se sacrificaba una mayor cantidad de animales, esto no solo proporcionaba más carne sino también más piel para la confección de pergaminos (Brinker-von der Heyde, 9-14). El desarrollo del papel en Europa (este ya se conocía en China y Corea), desde finales del siglo trece y sobre todo a partir del catorce, produjo importantes cambios en la producción y venta de libros, pero incluso después de 1450 se siguió empleando el pergamino para la edición de textos de especial valor como la Biblia.

Una lámina de pergamino consta a menudo de numerosos elementos que deben observarse todos juntos para comprender el concepto que se refleja en este. Lo primero es contemplar el texto, que a menudo aparece dispuesto en varias columnas. Las ilustraciones iniciales separan los distintos capítulos. Con frecuencia se marcaba de color el principio de línea, la mayoría de las veces en azul o rojo o se iba alternando. Numerosos manuscritos medievales aparecen con abundantes ilustraciones, lo que los convierten ya en grandes tesoros artísticos, que son por cierto casi los únicos que nos han llegado hasta hoy. La pintura al vidrio, los frescos - las esculturas no han sobrevivido a los avatares del tiempo. En cambio, los manuscritos son considerados como bienes culturales y poseen un valor a menudo incalculable. Por ello se les conserva con sumo cuidado en las bibliotecas y son incluso custodiados con cadenas para protegerlos ante posibles robos (como se puede ver hoy por ejemplo en la Biblioteca de la Iglesia de Santa Walburga en la ciudad holandesa de Zutphen). Muchos monasterios se intercambiaban los manuscritos para hacer copias y ocurría que a veces no volvían más a sus 
propietarios originales, lo cual parece sorprendente cuando se piensa en el inmenso valor de estos textos.

Con el paso del tiempo se traslada la producción de los manuscritos de los monasterios a las universidades y a los centros de escritura privados en las grandes ciudades. Pero también a las cortes de todo el mundo, donde se crean y reproducen una gran cantidad de textos, ya que los nobles en general poseían un elevado interés por la literatura cortesana, didáctica e instructiva. Tanto la poesía como la publicación de textos especializados (en Medicina, Política, Teología, Enseñanzas prácticas) gozaban de enorme prestigio, a pesar de que el oficio de escriba (incluso cuando trabajaba para un príncipe o rey) no estaba especialmente bien remunerado.

Coleccionar manuscritos era una de las pasiones de los grandes nobles y del clero, y desde que se desarrollan las universidades, a partir del siglo doce, se crean cada vez más bibliotecas privadas y el manuscrito pasa a convertirse en uno de los iconos centrales del Medievo. Por un lado, destacan por su cualidad como portadores de textos; con un adecuado tratamiento y conservación se impedía su deterioro incluso a lo largo de los siglos. Por otro lado, por su valor como medio para la mejor producción artística en la época antes de la Modernidad. Asimismo, la mayoría de los manuscritos medievales resultan documentos excepcionales de caligrafía. Es sorprendente la elevada calidad de estos textos si se piensa, entre otros, en lo áspero que era la superficie de escritura del pergamino a pesar de los mejores esfuerzos en confeccionar las láminas, o en la viscosidad de la tinta, que se producía normalmente del ácido tánico de agallas de roble, y donde se introducían plumas de ganso o pavo (Brown und Lovett).

Con el transcurso del tiempo se modifican tanto los tipos de escritura como las ilustraciones sin perjuicio de las distintas etapas de la historia de la lengua (en el caso de la lengua alemana: Alto alemán antiguo, Alto alemán medio y Alemán moderno) y de los contenidos. El manuscrito medieval se convertía así en un espejo central de la cultura de esa época, que superaba a la cultura oral todavía existente al permitir una mayor permanencia en la comunicación, la enseñanza y el entretenimiento. A través del manuscrito no sólo se conservan para la posteridad los mejores textos tanto ficcionales como científicos, sino asimismo los bienes culturales más esenciales de este tiempo. De hecho, las ilustraciones a mano de los manuscritos deberían considerarse, a pesar de su escaso tamaño, como las obras de arte más importantes de todo el Medievo.

\section{Literatura y manuscritos}


Por otra parte, el estado de transmisión de los manuscritos medievales nos ofrece valiosa información sobre la popularidad de algunas obras 0 autores. En todo caso conviene señalar que no siempre la cantidad es reflejo de la calidad, o a su vez, el juicio moderno sobre epopeyas o novelas cortesanas no necesariamente ha de coincidir con el de los lectores u oyentes del Medievo. El Libro de Héroes de Ambras contiene una serie de obras de extrema importancia de la literatura en Alto alemán medio, sin el cual probablemente no nos hubieran llegado. Pues en él se encuentran algunos textos como Kudrun o Mauritius von Craûn siendo los únicos que existen, a pesar de que ya se habían publicado a inicios o finales del siglo trece. Si bien estos se conservaron durante más de dos siglos a través de la oralidad, es de destacar el Libro de Héroes por ser el único testimonio escrito en el que se conservan dichos textos. Si no fuera por la labor de recopilación y escritura que llevó a cabo Hans Ried lo más seguro es que hubieran caído en el olvido. Aun cuando numerosas obras fueron asimismo (re)descubiertas en los siglos diecinueve y veinte, la gran mayoría de los manuscritos medievales se habría perdido.

En otros casos nos encontramos con un número inusualmente elevado de manuscritos con algunas obras que han sido reproducidas. Algunos de los ejemplos más importantes son libros muy conocidos, de renombre o quizás incluso "canónicos" como el anónimo Cantar de los Nibelungos, Parzifal y Willehalm de Wolfram von Eschenbach o Tristan de Gottfried von Straßburg. A esto hay que sumar incontables obras religiosas o didácticas, incluso también jurídicas, como Sachsenspiegel (El Espejo Sajón) de Eike of Repgow, que cuenta con 275 manuscritos. Si observamos algunas cifras de manuscritos llegaremos a un mayor conocimiento del estado de la cuestión.

De la obra Edelstein, escrita por Ulrich Bonerius en torno a 1350, se han conservado hasta el inicio del siglo dieciséis 36 manuscritos; de los Lieder de Walther von der Vogelweide $2 \mathrm{I}$ manuscritos bien con solo una parte o con el total de los textos; del Parzifal existen 88 manuscritos y 79 del Willehalm; en cambio su poema fragmentario Titurel solo se conserva en 3 manuscritos. Del Der arme Heinrich de Hartmann von Aue existen 7 manuscritos, 4 de su Erec, 33 del Iwein y 14 del Gregorius. De los Lieder de Neidhart se conservan 28 manuscritos; 9 del Der Welt Lohn de Konrad von Würzburg, 41 de su poema alegórico Die goldene Schmiede, 13 del Herzmære y 20 de su gran Trojanerkrieg.

$\mathrm{Si}$ echamos un vistazo a la literatura mística, entramos en una mayor categoría desde el punto de vista cuantitativo. Así por ejemplo de la obra de Heinrich Seuse Büchlein der ewigen Wahrheit nos han llegado 186 manuscritos; de su Exemplar en cambio tan solo hay 15, 14 de su Predigt y 16 de su Vita. De Das Licht der fließenden Gottheit de Mechthild von Magdeburg encontramos 19; de la obra en alemán de Revelationes de sacro exercitu virginum Coloniensum Elisabeth tenemos 9 manuscritos. Las cifras crecen considerablemente en la obras de Meister Eckhart. 
De su Predigt hay 82 manuscritos, 27 de su Reden der Unterscheidung, 2I de Spruchsammlung y 17 de su tratado Von abegescheidenheit. De la obra Karl der Große, escrita por Der Stricker, existen 42 manuscritos, 45 de su Kleinere Reimpaardichtungen, 13 de Pfaffe Amîs y 5 de Daniel von dem Blühenden Tal.

Todas estas cifras deben mirarse con gran atención, ya que las obras que gustaban más en su época, precisamente por su gran popularidad, podrían haber desaparecido rápido de las existencias, por lo cual hoy solo se habrían conservado unos pocos ejemplares. $O$ también podrían haber sido víctimas de las circunstancias bélicas, de incendios, inundaciones o de los bibliófilos. Numerosos manuscritos, como Der Ackermann aus Böhmen de Johannes von Tepl, del que hoy existen 17 ejemplares, se perdieron en la Guerras Husitas en el siglo quince, en las que los de Bohemios destruyeron sistemáticamente ciudades alemanas, iglesias y monasterios. Las obras de Johannes Hartlieb han sobrevivido relativamente bien a los avatares del tiempo, ya que existen 15 manuscritos de su De Amore deutsch, 20 del Alexander, 9 delsein Kräuterbuch, 10 del Kunst der Gedächtnüß , 7 del Mondwahrsagebuch, 10 del Namenmantik y 9 de su Secreta mulierum (para ver la totalidad de manuscritos de literatura medieval en lengua alemana, se puede consultar la siguiente página web: https://handschriftencensus.de/autoren\#B).

¿Qué nos dicen las cifras? En primer lugar se deben considerar de forma relativa, puesto que una mayor transmisión de manuscritos no se convierte de facto en una prueba segura del gusto por una obra concreta. Sin embargo, la probabilidad de que efectivamente haya sido el caso es al menos muy elevada. Al mismo tiempo, libros especializados como recetarios de cocina pueden haber sido leídos y suscitar pronto poco interés y esto habría afectado a otra clase de textos pragmáticos como por ejemplo de medicina. Si se atiende a la literatura especializada en la Edad Media, se observa una imponente cantidad de géneros como también de manuscritos (Haage und Wegner). Pero mucho más importante es que en la mayoría de los casos estos textos no son leídos por receptores individuales sino por un auditorio a través de una lectura en voz alta, ya que cualquier colectivo, ya se tratara de una corte, una comunidad monasterial o un público urbano, por no hablar de las universidades, prefería recibir una obra de forma recitada, lo que hacía que pudiera apreciarse y ser discutida en el grupo.

Por supuesto que nuestros juicios de hoy sobre textos concretos no tienen por qué coincidir necesariamente con los de aquellos de la Edad Media. Por el contrario, no sería desacertado tener en cuenta las cifras de los textos conservados, como ocurre por ejemplo en el caso del Parzifal de Wolfram von Eschenbach. La escasa recepción de su obra fragmentaria Titurel (solo hay 3 manuscritos) no significa, como han demostrado de forma insistente las recientes investigaciones, que tengamos que valorar poco el texto (Classen, Utopie, 1990). Mucho más tiene que ver con las circunstancias, las cuales no podemos reconstruir 
hoy de forma inequívoca. Un ejemplo nos lo muestra el Titurel de Albrecht von Scharfenberg, que desarrolló de forma extensa este breve texto de von Eschenbach y con el que alcanzó un gran éxito (de éste se han conservado 60 manuscritos).

Si se observa ahora la recepción de la poesía recitada, la situación cambia radicalmente. Los cantores, goliardos o similares se extendieron durante siglos de corte en corte, donde mostraban su repertorio para entretener al auditorio, bien a través del género de la poesía heroica o del lírica amorosa cortesana. Resulta difícil calcular hoy con exactitud el número de personas que acudía a sus recitaciones. Sin embargo podemos suponer que fue un número elevado el de las personas que, durante siglos, apreciaron sus obras. Del mismo modo, hay que pensar que cada manuscrito medieval constituye un tesoro para los bibliófilos, ya sea por empleo del pergamino, por su contenido, por las ilustraciones o por todo el texto en su conjunto. Poseer una gran biblioteca con manuscritos (quizás con un número de entre 200 y 300 ejemplares) conllevaba una distinción social y un aumento considerable de la percepción pública.

Mientras que en la Temprana Edad Media y Alta Edad Media la producción de manuscritos estaba principalmente limitada a los monasterios, con el paso de los siglos se secularizó este proceso y cada vez más aparecían nuevos grupos de jóvenes escribas que se interesaban por este oficio, cuando no trabajaban para la iglesia. Podemos identificar a partir del siglo trece una número creciente de centros, donde se crean intensamente manuscritos, lo que presuponía un una técnica de escritura altamente desarrollada, una capacidad artística y un conocimiento técnico y literarios excelentes. La diferencia entre los talleres de escritura religiosos y seculares se vuelve cada vez menos relevante, como ilustran por ejemplo los manuscritos del obispo Wolfger von Erla, donde, en torno a 1200, aparecieron las famosas versiones del Cantar de los Nibelungos.

Ya antes hay monjes en los monasterios que recogen por escrito la poesía popular, como el Beowulf, el Cantar de Hildebrand o el Heliand. En la Edad Media Tardía se continúa esta tarea en los talleres de escritura de las cortes principescas - de las ciudades, ya que utilizan el incipiente mercado del libro en dicha época como importante fuente de ingresos. El taller de Diebold Lauber (antes de 1427 después de 147I) en Hagenau (Alsacia) fue uno de los más productivos y exitosos de su tiempo, el cual se pudo mantener por un tiempo incluso después de la fase inicial de la introducción de la imprenta. En este taller se crearon más de 80 manuscritos con ilustraciones, que contenían algunas de las obras que más gustaban en la Edad Media (epopeyas, novelas cortesanas, epopeyas históricas) como el Tristan de Gottfried von Straßburg, el Karl der Große de Der Stricker, el Parzifal Wolframs von Eschenbach, el Wigalois de Wirnt von Gravenberg o el Trojanerkrieg de Konrad von Würzburg. A su vez, producían literatura mística, en busca de colmar los diferentes gustos y de aumentar así el número de clientes potenciales 
(Saurma, 200I; Fasbender, ed., 20I2). Esta estrategia comercial fue continuada por los primeros talleres de imprenta, ya que el mercado determinaba la demanda y con ello la producción tanto de manuscritos como de incunables o libros impresos.

\section{Los libros de horas: el punto culminante en la producción de manuscritos medievales}

Es casi imposible abarcar la diversidad de géneros que se encuentra en los manuscritos medievales, pero existe un amplio consenso, tanto en la investigación como entre el público, en que los libros de horas pertenecen a los verdaderos tesoros del arte del libro medieval (Classen, "The Book of Hours"). Presentaré al menos uno de ellos para que se puedan hacer una idea de las dimensiones estéticas, espirituales, literarias e histórico-artísticas que aquí se nos muestran. El Libro de Horas de María de Borgoña, que hoy se encuentra en la Biblioteca Nacional de Austria con la firma de Codex Vindobonensis 1857, fue creado en el último cuarto del siglo quince para una de las damas nobles más poderosas de la Edad Media (|457-|482), la hija del duque Carlos el Temerario, quien será la esposa del emperador Maximiliano I, hijo del emperador Federico III. Hasta su matrimonio, había vivido en Gante y disfrutaba de una vida lujosa, lo que explica que este libro, particularmente majestuoso, fuera creado para ella. La boda se pospuso unos meses debido al fallecimiento de su padre en la Batalla de Nancy en 1477, pero no se canceló. Se cree que María ya poseía para entonces este libro de horas, un regalo de su madrastra, Margarita de York, hermana del rey inglés Eduardo IV. Esta última se había casado con el duque Carlos en 1468, después de que muriera su primera esposa, Isabel de Borbón en 1465.

Los libros de horas, más conocidos bajo el nombre francés de "Livres d'heures", contenían en su mayoría los 150 salmos, oraciones y también calendarios, todo para "uso doméstico" y destinado principalmente a las damas principescas. Con la ayuda de estas joyas bibliófilas, relativamente pequeñas, la propietaria podía seguir el ritmo eclesiástico de la liturgia en privado y asimismo crear un espacio libre que le permitiese cumplir con sus obligaciones religiosas sin tener que salir de casa. Podría decirse mucho más sobre el contenido de este género, pero basta con destacar que el de María de Borgoña representa el ejemplo ideal, porque contiene prácticamente todos los elementos típicos. Su libro de horas supone además un punto culminante en la producción de los libros medievales (Delarun, ed.). La obra está compuesta, en su mayoría, por oraciones, un 
calendario, salmos, oficios y, asimismo, de ilustraciones a página completa o más pequeñas, principalmente de escenas bíblicas (Unterkircher).

Dondequiera que miremos, encontramos una infinidad de escenas de la vida cotidiana, de la naturaleza o del ámbito religioso. El llamado "horror vacui" es tan notable aquí como en el famoso coro de monjas del monasterio cisterciense de Wienhausen cerca de Celle (Classen, "The Medieval Monastery as a 'Gesamtkunstwerk'). Aunque los artistas ciertamente usaron una especie de muestrario para representar el sinfín de frutas, zarcillos, animales, interiores, actividades humanas (como un reflejo de las estaciones del año), pájaros y peces, aquí se observa de manera muy clara un nuevo sentido de la era medieval tardía donde se palpa el realismo. Al mismo tiempo, se descubre la gran alegría de los artistas, a quienes obviamente se les permitió dejar volar su imaginación e integraron grandiosas Marginalia, es decir, notas al margen.

Aunque se tratara de un libro de horas destinado a una dama de la nobleza, esto no impidió en absoluto que se insertara en el calendario un medallón (Medaillon) por cada página, que representaba una actividad rural típica de cada época del año. A su vez, se les permitió a los artistas integrar diversas escenas lúdicas, extrañas e incluso grotescas, cosa que era muy común en la Edad Media, ya fueran figuras humanas que emergían de las flores, bailarines y músicos, figuras desnudas que correteaban por el borde inferior, criaturas míticas $\circ$ incluso un ángel. El Rey David del Antiguo Testamento tocando su arpa aparece tantas veces como un niño desnudo agarrado a un andador. No había límite para la imaginación y lo que descubrimos en estos manuscritos ilustrados de finales de la Edad Media casi escapa a nuestro raciocinio (Camille; Classen, Hrsg., Imagination).

Desde el punto de vista de la Historia del Arte, se podría decir mucho de este manuscrito, magistralmente ilustrado, pero aquí nos basta con centrarnos únicamente en la fascinante imagen de una escena de interior. El espectador se ve inmediatamente atraído hacia el punto central dentro de una catedral gótica, donde la Virgen María, con una túnica de color azul oscuro, sostiene al Niño Jesús desnudo en su regazo. Varias damas de la corte, claramente en el estilo borgoñón de finales del siglo $\mathrm{XV}$, se arrodillan en señal de adoración a su lado derecho. Un hombre con una túnica roja oscura (Pluviale) se arrodilla al lado izquierdo de María. Toda la escena se abre ante nosotros a través de una ventana de vidrio redondo. Sin embargo, frente a la ventana, se sienta una dama, esplendorosamente ataviada, que sostiene un libro de horas sobre un paño verde y lo lee concienzudamente. Todo el cuadro está inundado de una luz que puede penetrar casi por completo, y sin obstáculos, a través de las ventanas góticas.

La perspectiva central que se utiliza aquí nos conduce a María directamente a través de la ventana, y de allí al altar en la parte posterior, dándonos el privilegio 
de participar directamente en los eventos sagrados de la iglesia. La mayoría de las investigaciones suponen que la figura femenina que aparece en el primer plano debe de ser Margarita de York, mientras que la dama arrodillada junto a María probablemente sea María de Borgoña, cuyas oraciones fueron respondidas directamente por la Virgen, (porque llegó a conocer personalmente al niño Jesús). Mediante la duplicación del espacio, separado por un gran ventanal, se consigue una transformación fascinante de la visualizacion del espectador, pues, por un lado, nos encontramos en el primer plano con un espacio privado dedicado a la devoción personal, y por otro lado, somos testigos de la visión divina de la mano de la dueña del libro de horas.

El diseño arquitectónico de la escena también demuestra ser extremadamente hábil y complejo, puesto que deambulamos por dos espacios con la mirada $y$, al mismo tiempo, nos encontramos como espectadores en nuestro propio tercer espacio. A medida que la dama del primer plano lee en su libro de horas, recibimos el mensaje de que su devoción realmente conduce a la transformación espiritual, y que ya no necesita los textos litúrgicos sino que se muestra muy cerca de la Virgen María. La ropa de la gente no podría ser más espléndida: en ella se refleja el decorado extremadamente lujoso de la Corte de Borgoña.

Los múltiples detalles (el perro faldero, el rosario, los cojines, dos lirios en flor en un jarrón, dos ángeles y todo el conjunto arquitectónico) sirven para alcanzar una inmediatez espiritual que limita con una visión mística y que aquí se ha plasmado figurativamente. No podemos saber con certeza si el artista trató de hacer reconocibles a las personas de manera individual, lo que sí está claro es que la situación religiosa no podría ser más enfática. Aunque ya aquí se aprecian los comienzos de la perspectiva central, toda la miniatura pertenece por completo a la historia cultural del gótico tardío $y$, por lo tanto, proporciona una evidencia irrefutable de que esta supuesta Edad Media no podría ser descrita de ninguna manera simplemente como "oscura". La integración de una gran cantidad de ilustraciones exactas de una amplia variedad de aves, frutas, plantas, instrumentos musicales y figuras humanas documenta claramente el alto nivel de comprensión y habilidad del arte que subyace en este libro de horas, en el que un fuerte sentido del realismo se combina con un profundo interés en la "transubstanciación" espiritual, un elemento característico de finales de la Edad Media (Classen, "DetailRealismus"; Smeyers, págs. 419-424; Fowler; Van Eyck), pero no solo podemos apreciar esto en los frescos góticos y en los altares grabados en madera, por ejemplo, en los de Tilman Riemenschneider, sino también en los manuscritos ilustrados de los siglos XIV y XV.

Aun así nos resulta imposible determinar con claridad la identidad de las dos figuras femeninas, por no hablar de la masculina, ya que el artista no tenía intención 
de ofrecer un retrato exacto de la mecenas (Margarita de York?). Sin embargo, esto no es sorprendente: el individuo todavía estaba sometido al poder divino y no debía abandonar este marco espiritual a través de la conciencia autobiográfica, de modo que tales retratos no se encuentran prácticamente por ninguna parte en la Edad Media. Este hecho no significa que no dispongamos de información alguna sobre los diversos poetas, porque incluso Hrotsvita von Gandersheim, y posteriormente Marie de France o Wolfram von Eschenbach, solo por nombrar unos cuantos, incluyeron muchas alusiones biográficas en sus textos.

\section{Codex Manesse}

Este anonimato intencionado es particularmente notable en el llamado Codex Manesse, creado entre 1280 y 1330 en nombre de la familia Manesse, proveniente de Zúrich, para salvar del olvido el gran tesoro del Minnesang en Alto alemán medio (Große Heidelberger Liederhandschrift). Este manuscrito consta de 426 hojas de pergamino y contiene los cantos de 110 poetas, casi todos ellos representados de cierta forma en iluminaciones de gran formato (un total de 137 miniaturas, II0 de las cuales forman la base), sin que ni siquiera una de ellas sea reconocible con precisión. Más bien nos encontramos con representaciones típicas e ideales que se remiten con frecuencia a uno de los contenidos de la canción y deben entenderse más de forma simbólica que biográfica (Brinker-von der Heyde, Pp. 8I-88; Voetz). Aquí también nos encontramos con un ejemplo revelador de la extensa empresa que representaba la producción de un manuscrito medieval, para la cual eran necesarios muchos especialistas y artistas diferentes, sin mencionar a los mecenas o donantes, sin los cuales no se habría podido crear ninguno de ellos. El Codex Manesse no nació de la noche a la mañana, sino que fue el resultado de esfuerzos colectivos a lo largo de los años, en los que los clientes pudieron reflejarse con orgullo.

\section{Oswald von Wolkenstein}

Conocemos otra gran colección de canciones de principios del siglo XV, pero que fue encargada por el propio poeta, Oswald von Wolkenstein (I376 / 77 1445). Hoy asumimos principalmente que este manuscrito B fue creado en el monasterio agustino de Neustift cerca de Brixen en el Tirol del Sur. Lo que llama la atención aquí como fenómeno singular es el retrato del poeta Oswald, que lo presenta de forma casi exagerada y vívida en plena realidad física, lo que ha llevado a varios investigadores a verlo como una obra que Oswald habría encargado al pintor renacentista italiano Antonio Pisanello, uno de sus alumnos (entre I 432 y 1438). El cuadro puede considerarse como uno de los primeros retratos auténticos 
de un poeta de la Edad Media alemana (Laussermeyer; Andergassen, págs. 79-8I), lo que hace que este manuscrito sea aún más valioso. Las partituras de las canciones también se pueden encontrar aquí en una etapa muy temprana, lo que convierte todo el códice en una obra de arte extraordinariamente valiosa que exige nuestro reconocimiento (http://www.literature.at/alo?objid=1049609). A partir de aquí podríamos incluir en la reflexión crítica innumerables manuscritos tardomedievales para apreciar con claridad el esplendor de estas obras artísticas, pero solo confirmaríamos lo que investigaciones previas ya han demostrado de manera extensa y convincente (Maestría medieval). Tanto el estilo como la elección del texto, la técnica de ilustración, los motivos de las miniaturas y otros elementos cambiaron con el tiempo. Cada vez surgieron más manuscritos con textos seculares, ya fueran literarios, científicos o didácticos. Los filósofos y teólogos escribieron obras extensas, los místicos registraron sus visiones, los médicos y arquitectos presentaron cada vez más sus hallazgos y conocimientos a un público que sabía leer y el número de ilustraciones en ellos resulta incalculable.

\section{Conclusiones}

Con esto llegamos al final de esta presentación y a nuestras conclusiones. El mundo medieval de los manuscritos en pergamino, y más tarde también de los manuscritos en papel, resultó ser sorprendentemente hábil, técnica y artísticamente; incluso demostrando poco interés por el realismo, especialmente en la fase temprana, todos los manuscritos medievales tienen en el fondo un extraordinario nivel artístico, caligráfico, literario además de un gran valor informativo. Las sumas de dinero invertidas en estas obras no se pueden comparar en absoluto con los precios de los libros modernos, porque eran tesoros de arte bibliófilo de primer orden. Los manuscritos no solo se utilizaron para preservar conocimientos o proporcionar información, o para servir como base para la misa (liturgia), ni fueron simplemente el medio para transmitir pensamientos poéticos, filosóficos, religiosos o técnicos. Mucho más allá fueron exponentes del arte representativo más exquisito, más perfecto y más valorado de su tiempo, y fueron diseñados con el mayor cuidado y, después almacenados, como podemos ver en algunas de las bibliotecas que aún existen de esa época.

Aquí es donde se percibe el verdadero esplendor de toda una época, porque los dueños de los manuscritos los custodiaban con sumo cuidado y otros los ansiaban tanto que se los apropiaban sin escrúpulos a través de su compra, pero también a través del robo, el hurto o el fraude. Desde entonces y hasta hoy, las bibliotecas de todo el mundo se han esforzado por adquirir, dentro de sus posibilidades, manuscritos medievales y añadirlos a su colección de tesoros repartida por todo el mundo (por ejemplo, el museo J. Paul Getty en Los Ángeles). 
Por supuesto, hay que decir que el gran aprecio por estas obras no se limitaba o limita a Europa (Toward a Global Middle Ages).

Teniendo todo esto en cuenta, la Edad Media debería dejar de parecernos un mundo bárbaro, primitivo y opresivo, y darnos, en su lugar, la impresión de un tiempo glorioso en el que, mediante el uso de manuscritos, se crearon las obras de arte más impresionantes de la historia de la humanidad. No tiene sentido ver la Edad Media como la "infancia" de nuestra propia época, en un sentido figurado (aun cuando incluso Dalarun sostiene este discurso, p. 7), porque es precisamente el impresionante testimonio de los manuscritos y sus ilustraciones, profundamente conmovedoras, lo que confirma que más bien somos nosotros quienes deberíamos ser considerados los bisnietos que, en cierto modo, hemos perdido la madurez intelectual, la profundidad espiritual y la capacidad artística de los premodernos. Por supuesto, esta opinión tampoco es del todo cierta, si bien podemos llegar a la conclusión, de que verdaderamente la enorme cantidad de manuscritos medievales son una prueba inequívoca de hasta qué punto esa época ya era desarrollada a este respecto $y$, por lo tanto, nuestra época actual no debería hacerle sombra. El valor puramente económico de estos manuscritos nos muestra hoy que se encontraban entre los mejores productos de su tiempo, y que por ello son totalmente dignos (de nuestro estudio y?) de nuestra admiración.

\section{Referencias bibliográficas}

ALTHOFF, Gerd: "Finsteres Mittelalter?! Zur Dekonstruktion eines Klischees." In: Farbe im Mittelalter: Materialität - Medialität - Semantik, hrsg. von Ingrid Bennewitz und Andrea Schindler. Akten des 13. Symposiums des Mediävistenverbandes vom I. bis 5. März 2009 in Bamberg. Bd. I. Berlin 20II, S. 47-63.

ANDERGASSEN, Leo: "Oswald von Wolkenstein und die Kunst: Selbstdarstellund und Repräsentation." In: Oswald von Wolkenstein: Leben - Werk - Rezeption, hrsg. von Ulrich Müller und Margarete Springeth. Berlin und New York 201 I, S. 77-88.

BEACH, Alison I.: Women as Scribes: Book Production and Monastic Reform in TwelfthCentury Bavaria. Cambridge Studies in Paleaography and Codicology. Cambridge 2004.

BECKER, Julia, Tino Licht, Bernd Schneidmüller: "Pergament." In: Michael Ott, Thomas Meier u. Rebecca Sauer, Hrsg.: Materiale Textkulturen. Konzepte Materialien - Praktiken. Materiale Textkulturen, I. Berlin, Boston und München 20I5, S. 337-347.

Brown, Michelle P. und Patricia Lovett: The Historical Source Book for Scribes. Toronto 1999. 
BUMKE, Joachim: Mäzene im Mittelalter: die Gönner und Auftraggeber der höfischen Literatur in Deutschland II50-1300. München 1979.

CAMILLE, Michael: Image on the Edge: The Margins of Medieval Art. London 1992.

ClASSEN, Albrecht: "The Book of Hours in the Middle Ages," Futhark: Revista de Investigación y Cultura 2 (2007): I I I-29.

: "The Book of Kells - The Wonders of Early Medieval Christian Manuscript Art Within a Pagan World," Mediaevistik 32 (2019; erschien 2020): 55-69.

: “'Detail-Realismus' im deutschen Spätmittelalter. Der Fall von des Strickers Daniel von dem blühen Tal und Konrads von Würzburg Turnier von Nantes," Studia Neophilologica 64 (1992): 195-220.

, Hrsg. Imagination and Fantasy in the Middle Ages and Early Modern Times: Projections, Dreams, Monsters, and Illusions. Fundamentals of Medieval and Early Modern Culture, 24. Berlin und Boston 2020.

: 'The Medieval Monastery as a 'Gesamtkunstwerk.' The Case of the 'Heideklöster' Wienhausen and Ebstorf.” In: Studi medievali XLIII, Fasc. II (2002): 503-34.

: Utopie und Logos. Vier Studien zu Wolframs von Eschenbach Titurel. Beiträge zur älteren Literaturgeschichte. Heidelberg 1990.

CORSTEN, Severin, Stephan FüsSEL und Günther PfLUG, Hrsg.: Lexikon des gesamten Buchwesens. Bd. 5. 2., völlig neubearbeitete Auflage. Hiersemann, Stuttgart 1999.

Delarun, Jacques, Hrsg. Das leuchtende Mittelalter. Aus dem Französischen von Birgit Lamerz-Beckschäfer. 3. Aufl. Darmstadt 20II (orig. 2002).

FOWLER, Alastair: Renaissance Realism: Narrative Images in Literature and Art. Oxford 2003.

GLENISSON, Jean, ed.: Le Livre au moyen âge. Turnhout 1988.

HAAGE, Bernhard Dietrich und Wolfgang WEGNER, unter Mitarbeit von Gundolf KEIL und Helga HAAGE-NABER: Deutsche Fachliteratur der Artes im Mittelalter und Früher Neuzeit. Grundlagen der Germanistik, 43. Berlin 2007.

EISENLOHR, Erika: "Die Kunst, Pergament zu machen." In: Uta Lindgren, Hrsg.: Europäische Technik im Mittelalter. 800 bis 1400. Tradition und Innovation. Ein Handbuch. Berlin 1996, S. 419-434.

FASBENDER, Christoph, Hrsg. Aus der Werkstatt Diebold Laubers. Berlin und New York 2012.

FüSSEL, Stephan: Johannes Gutenberg. 5. Aufl. Reinbek bei Hamburg 2019.

KLARER, Mario, Hrsg.: Kaiser Maximilian I. und das Ambraser Heldenbuch. Wien, Köln und Weimar 2019.

LaussermeYer, Maria Theresia: "Ist das Porträt Oswalds von Wolkenstein in Hs. B ein Werk Pisanellos?" In: Oswald von Wolkenstein: Beiträge der philologischmusikwissenschaftlichen Tagung in Neustift bei Brixen 1973, hrsg. von Egon 
Kühebacher. Innsbrucker Beiträge zur Kulturwissenschaft. Germanistische Reihe, I. Innsbruck 1974, S. 63-67.

Medieval Mastery: Book Illumination from Charlemagne to Charles the Bold, 8001475. General coordinator: Kris Callens. Leuven 2002.

Rural Space in the Middle Ages and Early Modern Age: The Spatial Turn in Premodern Studies, ed. Albrecht Classen, with the collaboration of Christopher R. Clason. Fundamentals of Medieval and Early Modern Culture, 9. Berlin und New York 2012.

SAURMA, Lieselotte E.: Spätformen mittelalterlicher Buchherstellung. Bilderhandschriften aus der Werkstatt Diebold Laubers in Hagenau. 2 Bde. Wiesbaden 200I.

SMEYERS, Maurits: Flemish Miniatures from the 8th to the Mid-16th Century: The Medieval World on Parchment. Leuven 1999.

Toward a Global Middle Ages: Encountering the World Through Illuminated Manuscripts, ed. Bryan C. KeENE. Los Angeles 2019.

UNTERKIRCHER, Franz: Das Stundenbuch der Maria von Burgund: Codex Vindobonensis 1875 der Österreichischen Nationalbibliothek. Kommentar. Glanzlichter der Buchkunst, 3. Graz 1993.

Van Eyck: eine optische Revolution, hrsg. Maximiliaan Martens, Till-Holger Borchert, Jan Dumolyn, Johan De Smet und Frederica Van Dam. Stuttgart 2020.

VOETZ, Lothar: Der Codex Manesse: Die berühmteste Liederhandschrift des Mittelalters. Darmstadt 2015.

Walther VON DER VOGELWEIDE: Leich, Lieder, Sangsprüche. I5., veränderte und um Fassungseditionen erweiterte Aufl. der Ausgabe Karl Lachmanns. Aufgrund der 14., von Christoph Cormeau bearbeiteten Ausgabe neu herausgegeben, mit Erschließungshilfen und textkritischen Kommentaren versehen von Thomas Bein. Berlin und Boston 2013.

Wolfger vON ERLA: Bischof von Passau (II9I-1204) und Patriarch von Aquileja (I204-|2|8) als Kirchenfürst und Literaturmäzen. Hrsg. von Egon BOSHOF und Fritz Peter Knapp. Germanische Bibliothek, 20. Heidelberg 1994. 